\title{
TWISTED LOCAL-PRODUCT MANIFOLDS
}

\author{
by S. A. ROBERTSON \\ (Received 17th January 1969)
}

This note is concerned with " twisted" analogues of the LP-structures (i.e. local-product structures) and grids of (4). To obtain these twisted structures, we modify the concepts of LP-structure and grid by removing the ordering of the local foliations involved in the definitions. The effect of this change is that global foliations need no longer exist, since the locally defined foils may now fit together to form self-intersecting immersed manifolds.

To each twisted LP-manifold $M$ we assign a homomorphism $h: \pi_{1}(M) \rightarrow S_{r}$ into the symmetric group $S_{r}$ of degree $r$, where $r$ is the number of foliations on $M$. Using $h$, we construct a covering $M^{*} \rightarrow M$ of $M$ by an untwisted LPmanifold $M^{*}$.

We extend the structure theorem for grids (4) to the twisted case, and apply this to the special case of complete flat manifolds. Nontrivial twisted grids exist on the flat Klein bottle, on the complete flat Möbius band, and on all but one (the torus $S^{1} \times S^{1} \times S^{1}$ ) of the ten compact flat 3-manifolds.

For simplicity, we restrict attention to twisted local-product structures of type $(k, \ldots, k)$. The definitions and theorems can be extended in an obvious way to include structures of type $\left(k_{1}, \ldots, k_{r}\right)$. Since we are ultimately interested in grids, we work with smooth (i.e. $C^{\infty}$ ) manifolds and maps.

\section{Twisted local-products}

Let $E$ and $F$ be finite-dimensional real linear spaces, and for any positive integers $r$ and $s$, let $E^{r}$ and $F^{s}$ denote the direct products $E \times \ldots \times E$ ( $r$ factors) and $F \times \ldots \times F$ ( $s$ factors) respectively. If $U$ and $V$ are open subsets of $E^{r}$ and $F^{s}$, then for all $i=1, \ldots, r$ and all $j=1, \ldots, s$ we put $U_{i}=p_{i}(U)$ and $V_{j}=q_{j}(V)$, where $p_{i}: E^{r} \rightarrow E$ and $q_{j}: F^{s} \rightarrow F$ are the $i$ th and $j$ th projections.

A smooth map $f: U \rightarrow V$ is called a twisted LP-map if there exists a map $\Pi:\{1, \ldots, r\} \rightarrow\{1, \ldots, s\}$ and for each $i=1, \ldots, r$ a smooth map $f_{i}: U_{i} \rightarrow V_{j}$, where $j=\Pi(i)$, such that $f_{i}\left(p_{i}(u)\right)=q_{j}(f(u))$ for all $u \in U$.

A twisted LP-transformation in $E^{r}$ is an invertible twisted LP-map $f: U \rightarrow V$, such that $U$ and $V$ are open subsets of $E^{r}$ and $f^{-1}$ is a twisted LP-map. Trivially, the set of all twisted LP-transformations in $E^{r}$ forms a pseudogroup. Thus a twisted LP-structure on a smooth $m$-manifold $M$ is a maximal atlas $\mathscr{M}$ of smooth charts $\xi_{a}: U_{a} \rightarrow U_{a}^{\prime}$ on $M$, where $U_{a}$ is an open subset of $E^{r}$ and $U_{a}^{\prime}$ is an open subset of $M$, indexed by some set $A$, such that for all $a, b \in A$, the map

$$
f_{a b}: \xi_{a}^{-1}\left(U_{a}^{\prime} \cap U_{b}^{\prime}\right) \rightarrow \xi_{b}^{-1}\left(U_{a}^{\prime} \cap U_{b}^{\prime}\right),
$$


given by $f_{a b}(u)=\xi_{b}^{-1}\left(\xi_{a}(u)\right)$, is a twisted LP-transformation. If $\operatorname{dim} E=k$, then $m=\operatorname{dim} M=r k$, and we say that $(M, \mathscr{M})$ is a twisted LP-manifold of type $k^{(r)}$.

Similarly, using LP-maps from open subsets of $E^{r}$ to open subsets of $F^{s}$, we can define the notion of LP-map from one LP-manifold modelled on $E^{r}$ to another modelled on $F^{s}$, which completes the construction of the twisted LPcategory.

\section{Untwisted coverings}

In this section, $(M, \mathscr{M})$ will denote a twisted LP-manifold of type $k^{(r)}$ modelled on $E^{r}$. First we construct, for each $x \in M$, a homomorphism $h_{p}: \pi_{1}(M, p) \rightarrow S_{r}$ into the symmetric group $S_{r}$ of degree $r$, as follows.

Let $\lambda: I \rightarrow M$ be a loop at $x$ in $M$, where $I=[0,1]$. Thus $\lambda(0)=\lambda(1)=x$. Then there is a sequence $\left(\xi_{1}, \ldots, \xi_{n}\right)$ of twisted LP-charts such that $\left\{U_{1}^{\prime}, \ldots, U_{n}^{\prime}\right\}$ covers $\lambda(I)$, where $\xi_{\alpha}: U_{(\alpha)} \rightarrow U_{(\alpha)}^{\prime}$, and $\lambda(] \frac{\alpha-1}{n}, \frac{\alpha}{n}[) \subset U_{(\alpha)}^{\prime} \quad(\alpha=1, \ldots, n)$. By definition, there exists a permutation $\Lambda_{(\alpha)}$ of the sequence $(1, \ldots, r)$ for each coordinate transformation $\xi_{\alpha+1}^{-1} \xi_{\alpha}$, with $\xi_{n+1}=\xi_{1}$. Trivially, the permutation $\Lambda=\Lambda_{1} \circ \ldots \circ \Lambda_{n}$ is independent of the choice of the charts $\xi_{\alpha}$, and is also independent of $\lambda$ up to homotopy rel. end-points. It is also immediate from the construction that if $\lambda_{1}$ and $\lambda_{2}$ are loops at $x$, and $\Lambda_{1}, \Lambda_{2}$ are the corresponding permutations, then the permutation $\Lambda_{1} \circ \Lambda_{2}$ corresponds to the loop $\lambda_{1} \lambda_{2}$.

Thus for each $x \in M$ there is a homomorphism $h_{x}: \pi_{1}(M, x) \rightarrow S_{r}$, given by $h_{x}[\lambda]=\Lambda$. Moreover, if $y \in M$ and $\gamma$ is any path in $M$ from $x$ to $y$, then $h_{y}=h_{x} \circ \gamma^{*}$, where $\gamma^{*}: \pi_{1}(M, y) \rightarrow \pi_{1}(M, x)$ is the isomorphism given by $\gamma^{*}[\mu]=\left[\gamma \mu \gamma^{-1}\right], \mu$ being any loop in $M$ at $y$. It follows that if $M$ is connected, then the homomorphism $h_{x}$ is independent of $x$ up to isomorphisms of $\pi_{1}(M, x)$, and we can write $h: \pi_{1}(M) \rightarrow S_{r}$, choosing some fixed $x \in M$ as base-point.

Now let $H: M^{*} \rightarrow M$ denote the regular covering of $M$ corresponding to the normal subgroup ker $h$ of $\pi_{1}(M)$. Thus $\pi_{1}\left(M^{*}\right)$ is isomorphic to ker $h$. Since any smooth immersion $f: M^{\prime} \rightarrow M$, where $\operatorname{dim} M^{\prime}=\operatorname{dim} M$, induces a twisted LP-structure $\mathscr{M}^{\prime}$ on $M^{\prime}$ from the given twisted LP-structure on $M$, we can give $M^{*}$ a twisted LP-structure $\mathscr{M}^{*}$ with respect to which $H$ is a twisted LP-covering. Thus $\mathscr{M}^{*}$ determines a homomorphism $h^{*}: \pi_{1}\left(M^{*}\right) \rightarrow S_{r}$. However, $h^{*}=h_{\circ} H^{*}$ where $H^{*}: \pi_{1}\left(M^{*}\right) \rightarrow \pi_{1}(M)$ is induced by $H$. Hence $h^{*}$ is trivial. Now there is an obvious " forgetful " functor $\Phi$ from the LP-category (of type $(k, \ldots, k)$ ) to the twisted LP-category, and $(N, \mathcal{N}) \in \mathrm{im} \Phi$ if and only if the homomorphism $\pi_{1}(N) \rightarrow S_{r}$ is trivial. In view of these remarks, it is natural to call $\left(M^{*}, \mathscr{M}^{*}\right)$ the smallest untwisted covering of $(M, \mathscr{M})$.

\section{Twisted grids}

Let $(M, \mathscr{M})$ be a twisted LP-manifold as before, and let $\rho$ be a smooth Riemannian metric on $M$. We say that $\mathscr{M}$ and $\rho$ are compatible, and call 
$\Gamma=(\mathscr{M}, \rho)$ a twisted grid on $M$, if $\mathscr{M}^{*}$ and $\rho^{*}=H^{*} \rho$ are compatible in the sense of (4), where $H:\left(M^{*}, \mathscr{M}^{*}\right) \rightarrow(M, \mathscr{M})$ is the smallest untwisted covering of $(M, \mathscr{M})$. Thus $\Gamma=(M, \rho)$ is a twisted grid on $M$ if and only if $\Gamma^{*}=\left(\mathscr{H}^{*}, \rho^{*}\right)$ is a grid on $M^{*}$.

To obtain a structure theorem for complete twisted grids (i.e. twisted grids $(\mathscr{M}, \rho)$ for which $\rho$ is complete), we have only to combine the smallest untwisted covering $H:\left(M^{*}, \Gamma^{*}\right) \rightarrow(M, \Gamma)$ with the universal covering

$$
K:\left(\tilde{M}^{*}, \tilde{\Gamma}^{*}\right) \rightarrow\left(M^{*}, \Gamma^{*}\right)
$$

and apply the structure theorem for grids. Now we can identify the universal covering $(\tilde{M}, \tilde{\Gamma})$ of $(M, \Gamma)$ with $\left(\tilde{M}^{*}, \tilde{\Gamma}^{*}\right)$, and put $J=H_{\circ} K$ for the universal covering map.

Let $\left\{M_{1}, \ldots, M_{r}\right\}$ be the foil-set of $\left(M^{*}, \Gamma^{*}\right)$ at some point $x \in M^{*}$. Then $(\tilde{M}, \tilde{\Gamma})$ is twisted grid-isomorphic to $\left(\underset{i=1}{\dot{X}} \tilde{M}_{i}, \Gamma^{\prime}\right)$, where $\Gamma^{\prime}$ is the standard trivially twisted grid on $\underset{i=1}{\dot{X}} \tilde{M}_{i}$ with respect to the product metric $\underset{i=1}{\dot{X}} \rho_{i}$, and $\rho_{i}$ is the metric induced on $\tilde{M}_{i}$ from $M^{*}$ by the inclusion.

It follows that $(M, \Gamma)$ is twisted grid-isomorphic to the quotient $\left(X \tilde{M}_{i}, \Gamma^{\prime}\right) / G$ of $\left(X \tilde{M}_{i}, \Gamma^{\prime}\right)$ by a discrete freely acting group $G$ of twisted grid-automorphisms. We note that the normal subgroup $G^{*}$ of $G$ of grid-automorphisms of the standard grid on $X \tilde{M}_{i}$ yields the smallest untwisted covering of $(M, \Gamma)$. That is, $\left(X \tilde{M}_{i}, \Gamma^{\prime}\right) / G^{*}$ is twisted grid-isomorphic to $\left(M^{*}, \Gamma^{*}\right)$.

Conversely, any such quotient is a twisted grid-manifold. In the next section, we give some simple examples of this construction.

\section{Twisted grids on flat manifolds}

To illustrate the above discussion, we examine a few simple examples of twisted grids on flat manifolds. These are particularly easy to handle, since the universal Riemannian covering of any complete flat $m$-manifold is isometric to Euclidean $m$-space $\boldsymbol{R}^{m}$ with its standard flat metric.

There are only five complete flat 2-manifolds: the Euclidean plane $E$ itself; the cylinder $C$; the torus $\boldsymbol{T}$; the Möbius band $\boldsymbol{M}$; and the Klein bottle $\boldsymbol{K}$ (see, for example, (5)). All admit untwisted grids, but only $\boldsymbol{K}$ and $\boldsymbol{M}$ possess nontrivial twisted grids (of type $1^{(2)}$ ). Figure 1 illustrates the twisted grid on $\boldsymbol{M}$. In this case, $M=R^{2} / Z$, where $Z$ acts on $R^{2}$ as a group of isometries generated by the glide-reflexion $g: R^{2} \rightarrow R^{2}$ given by $g(x, y)=(y+1, x+1)$.

The compact flat 3-manifolds have been determined by Hantzche and Wendt (3) (see also (1) and (5)). There are ten of these, and all but the torus $S^{1} \times S^{1} \times S^{1}$ admit nontrivial twisted grids. For example, we can represent one of these flat manifolds as the quotient $R^{3} / G$, where $G$ is the freely acting discrete group of isometries generated by the isometries $g_{i}: R^{3} \rightarrow R^{3}(i=1,2,3)$, 
where

$$
\begin{aligned}
& g_{1}(x, y, z)=(x+1, y, z) \\
& g_{2}(x, y, z)=(x, y+1, z) \\
& g_{3}(x, y, z)=\left(-\frac{1}{2} x+\frac{\sqrt{3}}{2} y,-\frac{\sqrt{3}}{2} x-\frac{1}{2} y, z+\frac{1}{3}\right)
\end{aligned}
$$

The lines $L_{1}, L_{2}$ and $L_{3}$ in $R^{3}$ generated by $(1,-1,0),(1,1,-2)$ and $(1,1,1)$ determine an untwisted grid on $R^{3}$ that is carried down to a nontrivial twisted grid on $R^{3} / G$. In fact, the image of the homomorphism $h: \pi_{1}\left(R^{3} / G\right) \rightarrow S_{3}$ is isomorphic to $Z_{3}$ in this case.

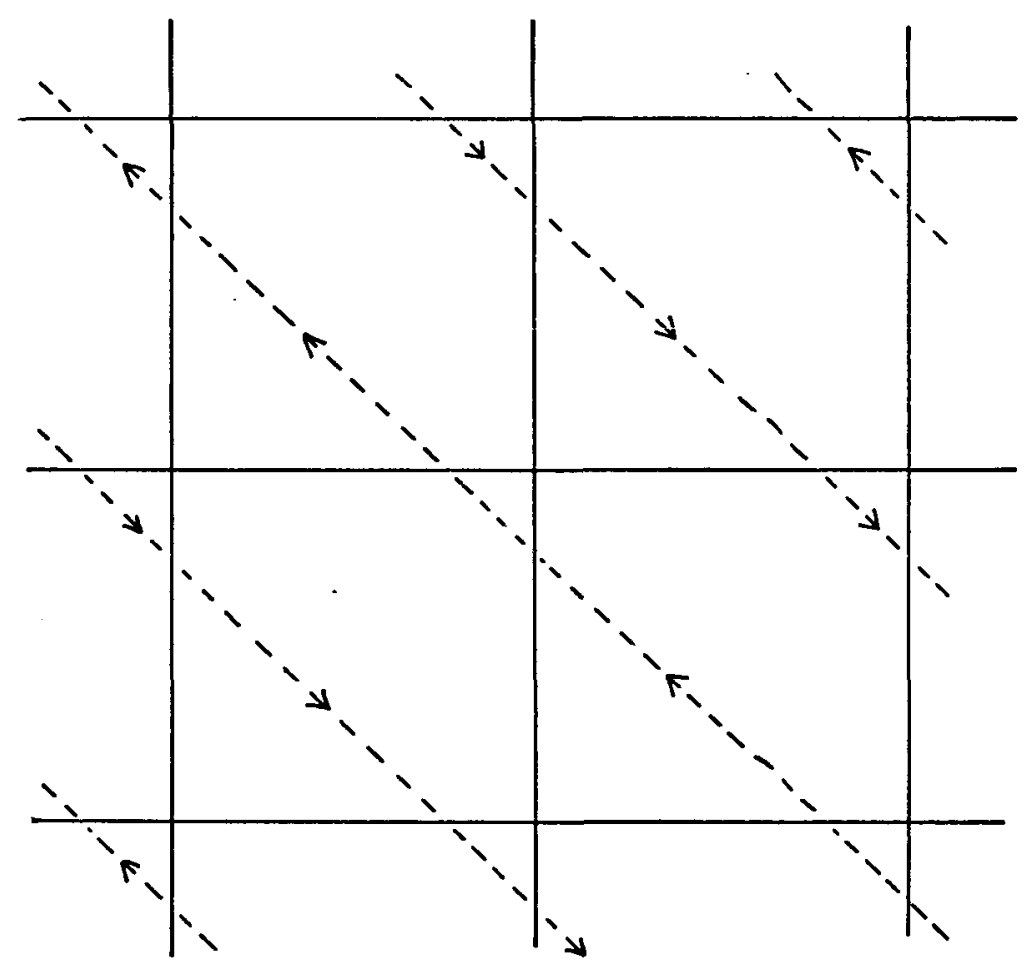

FIG. 1

\section{REFERENCES}

(1) L. S. Charlap, Compact flat riemannian manifolds I, Ann. of Math. (2) 81 (1965), 15-30.

(2) L. S. Charlap and A. T. Vasquez, Compact flat riemannian manifolds II, Amer. J. Math 87 (1965), 551-563. 
(3) W. Hantzche and H. Wendt, Dreidimensionale euklidische Raumformen, Math. Ann. 110 (1934-35), 593-611.

(4) S. A. Robertson, Grid manifolds, J. Differential Geometry (to appear).

(5) J. A. Wolf, Spaces of constant curvature (McGraw-Hill, New York, 1966).

Department of Pure Mathematics

THE UNIVERSITY

LIVERPOOL, 3 